\title{
LOS VALORES ÉTICOS Y SU RELACIÓN CON LA CIENCIA Y LA TECNOLOGÍA. UNA PROPUESTA DE TRABAJO EN EL AULA
}

\section{ETHICAL VALUES AND THEIR RELATIONSHIP WITH SCIENCE AND TECHNOLOGY. A PROPOSAL FOR WORKING IN THE CLASSROOM}

\author{
MYRIAM GARCÍA RODRÍGUEZ \\ garciamyriam09@gmail.com \\ Universidad de Oviedo - Grupo CTS \\ ORCID: 0000-0003-0086-6907
}

RECIBIDO: 29 DE SEPTIEMBRE DE 2019

ACEPTADO: 30 DE DICIEMBRE DE 2019

Resumen: El objetivo de este trabajo es presentar una propuesta didáctica para el aula de "Valores éticos", $1^{\circ}$ Ciclo de ESO, en el bloque correspondiente a "los valores éticos y su relación con la ciencia y la tecnología". Dicha propuesta está dirigida a la adquisición de una cultura científica crítica y responsable, consciente de los impactos sociales de la actividad tecnocientífica, sus potencialidades y sus riesgos, así como los interrogantes éticos que plantea. Para ello, se parte fundamentalmente del cine de ciencia-ficción como fuente para la reflexión filosófica. Los estudios sociales de la ciencia (estudios CTS) y la metodología de Filosofía para Niños $(\mathrm{FpN})$ son los fundamentos teóricos y prácticos de la propuesta. Tras hacer una breve referencia al marco teórico y metodológico del que se parte, se resume la secuencia de actividades que se propone. A partir de ahí, se presenta un ejemplo práctico para trabajar en el aula.

Palabras clave: valores éticos, cultura científica, filosofía para niños, estudios CTS

Abstract: The objective of this paper is to present a didactic proposal for the $1^{\circ}$ Cycle of Secondary School "Ethical values" subject, in the "ethical values and their relationship with science and technology" module. This proposal is aimed at the acquisition of a critical and responsible scientific culture, aware of the 
social impacts of the technoscientific activity, its potential and its risks, as well as the ethical questions it raises. To do this, it is essentially based on science fiction movies as a source for philosophical reflection. The social studies of science (STS studies) and the Philosophy for Children (P4C) program are the theoretical and practical foundations of the proposal. After making a brief reference to the theoretical and methodological framework, the sequence of activities proposed is summarized. Finally, a practical example for working in the classroom is presented.

Key words: ethical values, scientific culture, philosophy for children, STS studies

\section{Introducción}

La mayor parte de los analistas coinciden en que estamos adentrándonos en un modelo de sociedad realmente nuevo desde el punto de vista económico, político, social y cultural. Un aspecto significativo de este nuevo modelo de sociedad es la influencia que la ciencia y la tecnología ejercen en la conformación de múltiples aspectos de nuestra vida ${ }^{1}$ : el desarrollo científico-tecnológico no solo ha permitido sistemas de producción más perfeccionados que han alterado nuestras formas de relación con el medio, sino que también nos ha transformado a nosotros mismos, modificando las formas de relacionarnos, de trabajar, de actuar y hasta de pensar ${ }^{2}$.

Esta situación lleva aparejado una creciente preocupación institucional por el fomento de la cultura científica: el público debe

\footnotetext{
${ }^{1}$ En la medida en que se reconoce esta influencia de la ciencia y la tecnología en diversos aspectos de la dinámica social, son muchos los expertos que han acudido a alguno de los nuevos procesos de innovación científico-tecnológica para referirse al nuevo tipo de sociedad emergente: la "sociedad post-industrial" de Bell; la "tercera ola" de Toffler; la "sociedad red" de Castells; el "tercer entorno" de Echeverría, la "sociedad tecnológica global" de Queraltó, etcétera.

${ }^{2}$ TEZANOS, José Félix: "Los impactos sociales de la revolución tecnológica", en TEZANOS, José Félix: Los impactos sociales de la revolución científicotecnológica, Noveno Foro sobre Tendencias Sociales, Sistema, Madrid, 2007. Págs. 31-62.
} 
estar informado, conocer y comprender la ciencia y la tecnología de su tiempo. El Congreso Mundial sobre Ciencia celebrado en Budapest (Hungría) en julio de 1999, constituye un testimonio significativo de esta inquietud. Bajo el lema "Ciencia para el siglo XXI, un nuevo compromiso", el objetivo se centró en la búsqueda de nuevas vías para que los ciudadanos de todo el mundo pudieran tener acceso a los conocimientos científicos ${ }^{3}$. Un lenguaje parecido lo encontramos en los compromisos de las Metas Educativas $2021^{4}$, donde se reitera la necesidad de que los educadores contribuyan a que los ciudadanos y futuros profesionales adquieran una correcta percepción de los problemas y desafíos a los que se enfrenta hoy la humanidad, con el fin de participar en la toma fundamentada de decisiones y reorientar la investigación científica hacia la resolución de problemas.

Este argumento democrático es quizá el más ampliamente utilizado por aquellos que demandan un mayor fomento de la cultura científica. Más allá del valor intrínseco del conocimiento científico y tecnológico como parte de la herencia cultural de la humanidad, o de la necesidad de una fuerza de trabajo altamente cualificada, lo cierto es que una economía basada en el conocimiento científico y tecnológico requiere que una mayor proporción de ciudadanos comprenda, valore y actúe de manera crítica y responsable acerca de un número cada vez mayor de productos y servicios que incorporan nuevas tecnologías y avances científicos y médicos.

A estos objetivos se han dedicado numerosas investigaciones, currículos educativos y encuestas internacionales que contemplan

3 La Cumbre, convocada por la UNESCO y el Consejo Internacional para la Ciencia (ICSU), se clausuró con la aprobación por el plenario de una Declaración sobre la ciencia y el uso del saber cientifico (1999).

${ }^{4}$ ORGANIZACIÓN DE LOS ESTADIOS IBEROAMERICANOS - OEI: Metas educativas 2021. La educación que queremos para la generación de los bicentenarios, OEI-CEPAL-Secretaría General Iberoamericana, Madrid, 2010. 
la cultura científica como una de sus principales finalidades ${ }^{5}$. En el marco español, han sido variadas las iniciativas de mejora educativa que se han emprendido. En concreto, durante las últimas tres décadas se han desarrollado e implementado tres reformas educativas para las etapas previas a la formación universitaria: la Ley Orgánica de Ordenación General del Sistema Educativo (LOGSE) en 1990, la Ley Orgánica de Educación (LOE) en 2006 y la Ley Orgánica para la mejora de la calidad educativa (LOMCE) de 2013. Todas ellas han tratado de acercar la ciencia a la sociedad mediante la inclusión de asignaturas concretas en el currículo. Por ejemplo, "Ciencia, tecnología y sociedad" en la LOGSE, "Ciencias para el mundo contemporáneo" en el caso de la LOE, o la asignatura de "Cultura científica" en la LOMCE. Estas tres materias comparten un planteamiento abierto hacia el conjunto de la ciencia, lo que parece indicar que en las asignaturas tradicionales de ciencias no se agota todo lo que los ciudadanos podrían y deberían aprender sobre las relaciones entre la ciencia, la tecnología y la sociedad, sobre el papel de la ciencia en el mundo contemporáneo o sobre la cultura científica. No obstante, si bien la materia de Cultura Científica ha crecido en el número de horas y en los niveles en los que se imparte, las referencias a las relaciones entre el conocimiento científico-tecnológico y la sociedad -con énfasis en lo participativo y lo democrático- han disminuido drásticamente, sino desaparecido, con respecto a las anteriores ${ }^{6}$.

\footnotetext{
${ }^{5}$ Los trabajos de la American Association for the Advancement of Science (AAAS) o el programa PISA (Programme for International Student Assessment) de la OCDE, son representativos de esta preocupación creciente por la formación científica.

${ }^{6}$ MARTIN GORDILLO, Mariano: "Espacios curriculares para una ciencia cordial", en MARTÍN GORDILLO, Mariano y MARTINS, Isabel: Ciencia cordial. Un desafio educativo, Los Libros de la Catarata, Madrid, 2018. Págs. 15-29.
} 
En contrapartida, el nuevo currículo básico de la Educación Secundaria Obligatoria y del Bachillerato ${ }^{7}$ incorpora la materia de "Valores éticos" dentro del bloque de asignaturas específicas. La nueva materia, con un evidente enfoque crítico y reflexivo, incorpora entre sus contenidos un bloque específico sobre la dimensión moral de ciencia y tecnología en el que se contemplan aspectos tales como el problema de la tecnodependencia y la alienación humana, posibles aplicaciones inadecuadas en el terreno de la medicina y la biotecnología, el estudio de casos en los que la investigación científica no es neutral, sino determinada por intereses políticos, económicos, etc., o la necesidad de establecer límites éticos y jurídicos a la actividad tecnocientífica. De este modo, la materia de Valores Éticos se convierte en un complemento perfecto a la formación pura en ciencias, ya que permite introducir la reflexión ética y desarrollar en el alumnado un pensamiento crítico y cuidadoso sobre la actividad científicotecnológica y su influencia en el futuro. Evidentemente, todo esto se traduce en un aumento del esfuerzo docente por preparar materias nuevas y enfocar las clases de manera rigurosa pero atractiva.

El objetivo de este trabajo es presentar una propuesta didáctica para el aula de "Valores éticos", $1^{\circ}$ Ciclo de ESO, en el bloque temático correspondiente a "los valores éticos y su relación con la ciencia y la tecnología". Dicha propuesta está dirigida a la adquisición de una cultura científica crítica y responsable, consciente de los impactos sociales de la actividad tecnocientífica, sus potencialidades y sus riesgos, así como los interrogantes éticos que plantea. Para ello, se parte fundamentalmente del cine de cienciaficción como fuente para la reflexión filosófica. Los estudios sociales de la ciencia (estudios CTS) y la metodología de Filosofía

${ }^{7}$ MINISTERIO DE EDUCACIÓN, CULTURA Y DEPORTE - MECD: Ley Orgánica 8/2013, de 9 de diciembre, para la Mejora de la Calidad Educativa, Ministerio de Educación, Cultura y Deporte, Madrid, 2013. 
para Niños $(\mathrm{FpN})$ son los fundamentos teóricos y prácticos de la propuesta. Tras hacer una breve referencia al marco teórico, se describe la metodología didáctica a utilizar y la normativa vigente de la que parte. A continuación, se resume la secuencia de actividades que se propone $\mathrm{y}$, finalmente, se ofrece una ejemplificación de actividades para llevar a cabo en el aula.

\section{La metodología de Filosofía para Niños como estrategia didáctica para promover la cultura científica desde una perspectiva CTS. Hacia una educación STEAM \&Ethics}

Como ya se ha señalado anteriormente, el papel central de la actividad científica y tecnológica en todos los ámbitos de la vida pública y privada lleva aparejado una creciente preocupación institucional por la promoción de la cultura científica, diseñando e incorporando nuevas metodologías de aprendizaje a los currículos educativos.

Cada vez más oímos hablar de la educación STEM (Science, Technology, Engineering and Math), un enfoque pedagógico según el cual la Ciencia, la Tecnología, la Ingeniería y las Matemáticas forman un todo que interactúa y se afecta mutuamente. Las disciplinas no se enseñan de manera separada y aislada, sino integradas en proyectos interdisciplinarios, basados en situaciones del mundo real y orientados a la resolución de problemas prácticos. Ello requiere la puesta en marcha de metodologías de trabajo basadas en proyectos, prácticas de laboratorio y herramientas tecnológicas. Entre los objetivos principales de la educación STEM se incluye el fomento de las vocaciones en ciencias, el desarrollo de habilidades científicas como la curiosidad, la capacidad de análisis, la investigación o la resolución de problemas (PBL). Recientemente, el modelo STEM ha evolucionado hacia un nuevo paradigma educativo que plantea la integración de las artes en el 
marco de las disciplinas científicas, dando lugar a la educación STEAM (Science, Technology, Engineering, Arts and Math), que enfatiza la importancia de las artes plásticas y el diseño, la creatividad y la innovación. Lo que se obtiene es un nuevo marco de aprendizaje donde la curiosidad y el interés personal se convierten en el punto de partida para la exploración de diferentes soluciones, fomentando la imaginación y los procesos creativos, y prestando especial atención a planteamientos interdisciplinares y colaborativos ${ }^{8}$.

Este nuevo enfoque no solo pone en cuestión un sistema educativo basado, fundamentalmente, en la separación histórica entre humanidades y ciencias, sino que facilita el aprendizaje de las destrezas y habilidades que los estudiantes necesitan para afrontar la complejidad de la realidad contemporánea, lo que demuestra que una educación integral para el mundo contemporáneo requiere constantemente de enfoques renovados de investigación y de innovación educativa. Este trabajo se basa en esta convicción y pretende defender la conveniencia de incorporar un nuevo modelo educativo que apunte no solo al pensamiento crítico y creativo, sino también al pensamiento cuidadoso, desarrollando en los estudiantes habilidades éticas, políticas y de compromiso social. El modelo STEAM \&Ethics.

Si atendemos a las recomendaciones del Parlamento Europeo y del Consejo de la Unión Europea sobre las competencias clave para el aprendizaje permanente ${ }^{9}$, según las cuales las competencias básicas en ciencia y tecnología "deberán permitir a cada persona

\footnotetext{
${ }^{8}$ Véase, al respecto, SOUSA, David y PILECKI, Tom: From STEM to STEAM: Using Brain-Compatible Strategies to Integrate the Arts, CA: SAGE, Thousand Oaks, 2013.

9 PARLAMENTO EUROPERO Y CONSEJO DE LA UNIÓN EUROPEA: Recomendación 2006/962/CE del Parlamento Europeo y del Consejo, de 18 de diciembre de 2006, sobre las competencias clave para el aprendizaje permanente, Diario Oficial de la Unión Europea L 394/10, Bruselas, 2006.
} 
comprender mejor los avances, las limitaciones y los riesgos de las teorías científicas, las aplicaciones y la tecnología en las sociedades en general (en cuanto a la toma de decisiones, los valores, las cuestiones morales, la cultura, etc.)", entonces, la importancia de una educación STEAM \&Ethics es incuestionable.

Una tesis comúnmente aceptada es que la educación científica debe estar orientada a preparar a los estudiantes como si todos pretendieran llegar a ser especialistas en biología, física o química, y que el profesorado debe estar preparado para seleccionar y formar a esa minoría de futuros científicos. En consecuencia, los currículos plantean como objetivos prioritarios que los estudiantes conozcan, fundamentalmente, los conceptos, principios y leyes de esas disciplinas. Sin embargo, en la medida en que el desarrollo científico-tecnológico se convierte en motivo de conflictos sociales que involucran aspectos políticos, económicos y éticos, es necesario también que los educadores contribuyan a que los ciudadanos y futuros profesionales aprendan a integrar la información recibida y hacer uso de la reflexión crítica en un intento por formar juicios independientes sobre asuntos controvertidos o al tomar decisiones cotidianas como usuarios y consumidores. Las polémicas en torno a la seguridad para la salud de los transgénicos o el movimiento antivacunas pueden ser vistos como ejemplos de este fenómeno de politización del desarrollo científico-tecnológico. Cabe esperar, además, que una cultura científica crítica y responsable contemple no solo los conocimientos básicos de la "ciencia ortodoxa", sino también contenidos relativos a los riesgos e incertidumbres, efectos adversos, usos políticos, dilemas éticos o influencias económicas de la investigación científica y el desarrollo tecnológico ${ }^{10}$.

10 Véase, al respecto, DÍAZ, Irene y GARCÍA, Myriam: "Más Allá del Paradigma de la Alfabetización. La Adquisición de Cultura Científica como Reto Educativo", en Formación Universitaria, Vol. 4 N. ${ }^{\circ}$ 2, Chile, 2011. Págs. 3-14. 
En resumen, se considera que una cultura científica crítica y responsable no se limita a responder correctamente a las preguntas habituales de las materias científicas, sino que incluye también conocimientos, valores y un fuerte componente práctico; esto es, la generación de actitudes y pautas de comportamiento. Por ejemplo, tener el hábito de hacer uso de la información científica a la hora de tomar decisiones, tanto en situaciones ordinarias como extraordinarias: leer los prospectos de los medicamentos, prestar atención a las etiquetas de los alimentos, tener en cuenta la opinión médica al seguir una dieta o tratar de mantenerse informado ante una alarma sanitaria -como la reciente alerta por listeriosis en España o la crisis de los incendios en el Amazonas- ${ }^{11}$.En este sentido, el proceso de enculturación científica no puede ser contemplado como una mera transmisión de conocimientos, sino como la generación de aprendizajes transferibles a contextos reales, tomando conciencia del papel central que juegan la ciencia y la tecnología en temas como la salud, los recursos alimenticios y energéticos, la conservación del medio ambiente o la comunicación. Se trata, en definitiva, de incluir la dimensión éticapolítica en la educación STEAM y, por extensión, las disciplinas filosóficas que tradicionalmente han sido las encargadas de desarrollar y fomentar en el alumnado un pensamiento crítico y autónomo.

La materia de Valores Éticos, tal y como aparece recogido en el Anexo II para las Materias del Bloque de Asignaturas Específicas, puede servir como espacio privilegiado para potenciar la autonomía de los estudiantes y su capacidad de elaborar libre y racionalmente un pensamiento propio, de manera que puedan ejercer progresivamente su libertad y responsabilidad de modo consciente,

${ }^{11}$ Véase, al respecto, CÁMARA HURTADO, Montaña y LÓPEZ CEREZO, José Antonio: "Dimensiones políticas de la cultura científica, en LÓPEZ CEREZO, José Antonio y GÓMEZ GONZÁLEZ, Francisco Javier: Apropiación social de la ciencia, Biblioteca Nueva-OEI, Madrid. Págs. 63-89. 
crítico, reflexivo y fundamentado en valores éticos. En concreto, la inclusión de un bloque dedicado a "los valores éticos y su relación con la ciencia y la tecnología" se orienta hacia el análisis de "la dimensión moral de la ciencia y la tecnología, evaluando el impacto positivo y negativo que estas pueden tener en todos los ámbitos de la vida humana (social, económico, político, ético, ecológico...)" así como hacia el reconocimiento de "la necesidad de poner límites éticos y jurídicos a la investigación y práctica científica y tecnológica" ${ }^{92}$. Son las cuestiones propias del enfoque CTS, como ¿Es lo mismo progreso humano que progreso tecnológico? ¿Todo lo que se puede hacer tecnológicamente, se debe hacer? ¿Es el científico socialmente responsable de su actividad? ¿Qué papel pueden jugar los ciudadanos?, etcétera.

Frente a la visión tradicional de la ciencia como una actividad eminentemente teórica, objetiva, racional y autónoma, al margen de los contextos en los que se desarrolla, los estudios CTS ponen el énfasis en la dimensión social de la actividad científica y proponen entenderla como una actividad inherentemente social, donde los valores, los intereses, las luchas de poder y los grupos de presión desempeñan un papel decisivo en la producción y aceptación de una teoría científica o de un artefacto tecnológico. El punto de inflexión lo marca Thomas Kuhn cuando propone estudiar la ciencia desde una perspectiva sociohistórica que atienda no solo a valores epistémicos sino también no epistémicos o extra-científicos -por ejemplo, valores económicos, políticos, sociales, jurídicos, ecológicos o militares-. Siguiendo la estela kuhniana, autores de la nueva sociología de la ciencia, como David Bloor, Harry Collins, Wiebe Bijker o Bruno Latour, ayudaron a desmitificar la imagen triunfalista e idealizada de la ciencia. Sus estudios pusieron de

${ }^{12}$ MINISTERIO DE EDUCACIÓN, CULTURA Y DEPORTE - MECD: Real Decreto 1105/2014, de 26 de diciembre, por el que se establece el currículo básico de la Educación Secundaria Obligatoria y del Bachillerato, Ministerio de Educación, Cultura y Deporte, Madrid, 2014. 
manifiesto que la ciencia resulta una actividad mucho más costosa, lenta, frustrante y difícil de lo que la literatura tradicional muestra. Asimismo, las últimas investigaciones de autores como Paul Durbin, Sheila Jassanoff, Carl Mitcham o Langdon Winner, centradas en la gestión y evaluación del desarrollo científicotecnológico, cuestionan la vieja imagen de la ciencia como actividad que conduce directamente hacia mejores productos para un mejor bienestar y comparten el compromiso por un estudio más crítico que denuncia los efectos negativos y las consecuencias éticas, sociales y medioambientales de una ciencia sin control ${ }^{13}$.La ciencia no es algo incuestionable, acabado y cerrado. Es preciso pensar y presentar la ciencia y sus aplicaciones como algo problemático, abierto, revisable y criticable, que invita a la discusión y a la clarificación.

Por todo ello, el enfoque CTS se revela como una alternativa que puede ser extremadamente efectiva para ofrecer al alumnado una aproximación a la ciencia y la tecnología más realista y vinculada con la cotidianidad de trabajo científico, más crítica y más responsable respeto al cambio científico-tecnológico. Dicho aprendizaje se ve muy favorecido cuando el alumnado tiene la oportunidad de pensar y argumentar sobre la ciencia en un contexto controlado de participación.

La metodología de FpN desarrollada por Mathew Lipman y sus colaboradores es quizá una de las más adecuadas para esto, ya que uno de sus aspectos más novedosos reside en el uso de una metodología dialógica que favorece la participación de los

\footnotetext{
${ }^{13}$ Para una panorámica general del enfoque CTS véase, al respecto GONZÁLEZ GARCÍA, Marta, LOPEZ CEREZO, José Antonio y LUJÁN, José Luis: Ciencia, tecnología y sociedad: una introducción al estudio social de la ciencia y la tecnología, Tecnos, Madrid, 1996.
} 
estudiantes en la construcción colectiva del conocimiento ${ }^{14}$. Su propuesta de transformar el aula en una comunidad de investigación y sus aportaciones con respecto al origen social del pensamiento permiten desarrollar un proyecto educativo basado en la mejora de las habilidades de pensamiento. Será a través del diálogo filosófico y del perfeccionamiento de las estrategias de razonamiento, argumentación y fundamentación de ideas, creencias y valores, como se irá mejorando la manera de pensar. En realidad, no se trata más que de aplicar el diálogo socrático, pero enriquecido por la psicología cognitiva y social de George Mead, Jerome Bruner y Lev Vygostky, la propuesta del pragmatismo norteamericano de Charles Peirce y John Dewey; y, en desarrollos posteriores de otros autores continuadores del programa, los planteamientos del aprendizaje significativo, especialmente los trabajos de Paulo Freire.

En primer lugar, se recuperan las enseñanzas socráticas, donde la práctica filosófica no consiste en el postulado de teorías abstractas ni en la exégesis de textos antiguos, sino que implica el cuestionamiento o la problematización de un tema o situación que nos interpela de un modo u otro. La finalidad no es la derrota del oponente, sino llegar a un consenso o acuerdo acerca de las cuestiones tratadas. En este sentido, se distingue el diálogo socrático de una mera conversación -donde no hay forzosamente un propósito- y de un discurso persuasivo -donde se intenta manipularDe ahí que el docente deba renunciar al rol tradicional de "experto", visto como figura de autoridad, y asuma la función de mediador mayéutico, ayudando a estimular el intercambio de opiniones, ideas y puntos de vista.

Para esta tarea, se insiste en una determinada manera de entender la práctica en el aula, la práctica de convertirla en una comunidad de

${ }^{14}$ Para una introducción completa al programa de $\mathrm{FpN}$, véase al respecto LIPMAN, Matthew, SHARP, Ann y OSCAYÁN, Frederick: La filosofia en el aula, Ediciones de la Torre, Madrid, 1992. 
reflexión formada por individuos comprometidos con la exploración de temas complejos. El objetivo no es tanto la transmisión de conocimiento preciso e inequívoco mediante la absorción de datos e información provenientes del docente, sino el aprendizaje por descubrimiento basado en la resolución de problemas en un ambiente de indagación donde los estudiantes puedan manejar información relevante, intercambiar opiniones, articular conocimientos $\mathrm{y}$ formular argumentos $\mathrm{y}$ contraargumentos. Ideas que ya están presentes en el constructivismo social de Bruner y Vygostky. En lugar de concebir la mente como un recipiente pasivo y vacío que debe ser rellenado con información y contenidos meramente teóricos, se presupone que el alumnado aprende al estar involucrado de manera activa a través de la interacción con el ambiente $\mathrm{y}$ resolviendo problemas relacionados con su realidad próxima.

Se llega entonces a "la comunidad de investigación", un concepto adoptado por Peirce y que consiste fundamentalmente en aplicar los mismos procesos de la investigación científica a la práctica educativa. "De la misma forma en que los científicos aplican el método científico a la exploración de las situaciones problemáticas, los estudiantes deberían hacer lo propio si quieren aprender a pensar por sí mismos" ${ }^{\prime 15}$. En esa exploración conjunta, se sustituye la competitividad por el esfuerzo conjunto. El diálogo, el respeto mutuo y el razonamiento de las propias opiniones son prácticas que terminan convirtiéndose en el modo normal de conducta de los/as estudiantes y el docente. Se trata, en última instancia, de seguir la ruta trazada por Dewey y su insistencia en una educación democrática, entendiendo la democracia no en sentido formal o institucional, sino como forma de vida y de organización individual y social. El proceso educativo ya no consiste en decirle al sujeto

\footnotetext{
${ }^{15}$ LIPMAN, Mathew: Pensamiento complejo y educación, Ediciones de la Torre, Madrid, 1998. Pág. 57.
} 
que aprende lo que debe o no debe hacer, sino en hacerle partícipe y artífice de su propia vida, tomando conciencia de la realidad que le rodea y comprometiéndose de forma crítica con su transformación. Tal es la propuesta de Freire y su pedagogía de la autonomía.

En este punto cabe señalar, de acuerdo con los planteamientos del aprendizaje significativo, que los estudiantes se sentirán mucho más dispuestos a participar en un diálogo en el que se puedan plantear problemas parecidos a los suyos, temas que les interesen o les interpelan de algún modo. Algo que tradicionalmente se ha hecho partiendo de un relato, un cuento o una fábula. De ahí que Lipman y sus colaboradores propongan el uso de novelas con protagonistas que viven situaciones con las que los estudiantes se puedan identificar. Ello implica conocer los saberes previos de los estudiantes, sus intereses e inquietudes, a fin de conseguir que se impliquen en el aula y se apropien de los contenidos que allí se tratan para su uso en la vida diaria.

La propuesta de este trabajo es reivindicar el cine como recurso susceptible de una lectura filosófica que se adapte mejor a la era pantallística y audiovisual que transitan hoy los jóvenes. Así, al "leer" una película de cine como texto fílmico podremos rastrear la presencia de cuestiones típicamente filosóficas, vinculando la reflexión teórica con la experiencia existencial de cada uno/a. El valor de esta práctica no reside tanto en las respuestas que pueda proporcionar como en la búsqueda de significados que nos ayuden a comprender el mundo que nos rodea.

Evidentemente, todo esto se traduce en un aumento del esfuerzo docente por preparar materiales nuevos y enfocar las clases de manera rigurosa pero atractiva. Con el fin de aportar una guía práctica para el docente y mostrar la viabilidad de la propuesta se describe, a continuación, la metodología didáctica a utilizar y se ofrece un breve catálogo de películas para, finalmente, ejemplificar una sesión en el aula. 


\section{El uso de la ciencia ficción como estrategia didáctica para el aula de Valores Éticos}

De acuerdo con el Anexo II sobre Orientaciones para facilitar el desarrollo de estrategias metodológicas que permitan trabajar por competencias en el aula, de la Orden ECD/65/2015, el actual proceso de inclusión de las competencias como elemento esencial del currículo supone optar por "metodologías activas y contextualizadas" que faciliten "la participación e implicación del alumnado y la adquisición y uso de conocimientos en situaciones reales". Por un lado, las metodologías activas deben apoyarse en "estructuras de aprendizaje cooperativo", orientadas a la "resolución conjunta de las tareas" de forma que "los miembros del grupo conozcan las estrategias utilizadas por sus compañeros y puedan aplicarlas a situaciones similares". Para ello, las "estrategias interactivas" son vistas como "las más adecuadas" al permitir "compartir y construir el conocimiento y dinamizar la sesión de clase mediante el intercambio verbal y colectivo de ideas". Por otro lado, las metodologías que contextualizan el aprendizaje "favorecen la participación activa (...) así como la motivación de los alumnos y alumnas". Para ello, se recomienda la realización de actividades orientadas a la resolución de tareas, proyectos o situaciones-problema potencialmente reales ${ }^{16}$. En cuanto a las recomendaciones metodológicas para la materia de Valores Éticos, dado el carácter esencialmente instrumental de los valores éticos para la vida diaria, el currículo básico de la Educación Secundaria Obligatoria y del Bachillerato hace especial hincapié en la incorporación de estrategias encaminadas a la

${ }^{16}$ MINISTERIO DE EDUCACIÓN, CULTURA Y DEPORTE - MECD: Orden $E C D / 65 / 2015$, de 21 de enero, por la que se describen las relaciones entre las competencias, los contenidos y los criterios de evaluación de la Educación Primaria, la Educación Secundaria Obligatoria y el Bachillerato, Ministerio de Educación, Cultura y Deporte, Madrid, 2015. Pág. 17 
elaboración de productos "socialmente relevantes" donde el alumnado pueda comprometerse activamente en la transformación de su entorno, garantizando así la transmisión de ese aprendizaje a la vida individual y social.

Por todo ello, y atendiendo a las recomendaciones de la normativa vigente, la metodología propuesta en este trabajo es fundamentalmente activa y participativa, favoreciendo la implicación del alumnado en la construcción de los aprendizajes y en la adquisición de las competencias. Se prescinde de las lecciones magistrales y se opta por una metodología dialógica, inspirada en el proyecto de FpN. En la búsqueda de recursos audiovisuales que fomenten el diálogo, el cine de ciencia-ficción aparece como un excelente recurso didáctico para motivar la discusión y el intercambio de opiniones entre el alumnado.

Basta con pasearse por un aula de Secundaria o Bachillerato para darse cuenta de que el cine de ciencia-ficción es uno de los medios de entretenimiento más apreciados por los adolescentes. No obstante, sirvan como muestra los siguientes datos. Según el último estudio realizado por el Ministerio de Cultura en su Anuario de Estadísticas Culturales, acudir al cine es una de las actividades culturales realizadas con mayor frecuencia por la población española, con tasas de asistencia anual del $54 \%$, siendo los menores de 25 años los que más acuden a las salas ${ }^{17}$. Al menos 7,4 de cada 10 va una vez al mes ${ }^{18}$.En un estudio anterior sobre hábitos de consumo cinematográfico elaborado por Cinesa, el género cinematográfico preferido por los encuestados es la ciencia ficción

17 MINISTERIO DE EDUCACIÓN, CULTURA Y DEPORTE - MECD: Anuario de Estadísticas Culturales 2018, Secretaría General Técnica, Subdirección General de Atención al ciudadano, Documentación y Publicaciones, Ministerio de Educación, Cultura y Deporte, Madrid, 2018.

18 FEDERACIÓN DE ENTIDADES DE EMPRESARIOS DE CINE DE ESPAÑA - FECE: Salas de cine. Datos y cifras 2018, FECE, Madrid, 2019. 
$(19 \%)^{19}$. No se puede negar, por tanto, que el cine cumple con el requisito de fomentar el interés y la motivación del alumnado. Como forma de entretenimiento que busca sorprender y emocionar puede ser una vía útil para generar actitudes positivas en el aula. Por otro lado, es importante tener en cuenta que el cine no es simplemente un medio de diversión y distracción, sino que constituye fundamentalmente un poderoso instrumento de transmisión y configuración de actitudes, valores y pautas de comportamiento. Este hecho puede ser visto como un problema o como una oportunidad. Precisamente por su carácter de medio de comunicación de masas, su cometido abarca desde la información hasta la persuasión y el adoctrinamiento, creando representaciones del mundo y de la realidad acríticas o estereotipadas que modulan, de forma consciente o inconsciente, las conductas de los espectadores $^{20}$. Por todo ello, es necesaria una formación en el medio para que el espectador pueda adoptar una postura crítica y activa ante el mensaje ${ }^{21}$. Ahí reside el valor educativo y cultural del cine como estrategia didáctica. Al transmitir ideas, valores, modelos de conductas, estilos de vida o referentes ideológicos, el cine ofrece la posibilidad de utilizar sus productos para desarrollar en los estudiantes competencias de reflexión y espíritu crítico, mostrando las diferencias y semejanzas entre los contenidos que se imparten en el aula, los que ficciona el cine y los que se vivencian en la realidad.

Sin embargo, si bien existen algunos ejemplos sobre la utilización del cine como recurso didáctico en el aula de Primaria y Secundaria, el uso de la ciencia ficción aún no está suficientemente

19 COMPAÑÍA DE INICIATIVAS Y ESPECTÁCULOS S.A - CINESA: Hábitos de consumo cinematográfico en España, Cinesa, Madrid, 2017.

${ }^{20}$ CABERO, Julio: "Educación en valores y cine", en Making of: Cuadernos de Cine y Educación, N. ${ }^{\circ}$ 20, Barcelona, 2003. Págs.16-30.

${ }^{21}$ AMAR, Víctor: Comprender y disfrutar el cine. La gran pantalla como recurso educativo, Grupo Comunicar, Huelva, 2003. 
explotado como recurso para motivar la reflexión sobre la visión del mundo científico que transmite. ¿Cómo son las personas que trabajan en ciencia? ¿En qué medida son responsables de los avances tecnológicos?¿Cómo influyen esos avances en nuestra sociedad? etc. La visión puede ser utópica o catastrofista, ficcionando otros mundos posibles en los que la ciencia se identifica o bien como salvadora o bien como devastadora del mundo. Se suelen representar sociedades futuras tecnológicamente avanzadas donde predomina la confianza en las posibilidades del desarrollo científico como garante de bienestar, pero también es común encontrar alusiones a los riesgos de la actividad científicatecnológica, como la contaminación, el cambio climático, el agotamiento de los recursos, el armamento nuclear o la eugenesia positiva. De este modo, la ciencia ficción permite introducir temas de anticipación y plantear una reflexión ética acerca de los desarrollos científico-tecnológicos y sus consecuencias: cómo se hace ciencia, para qué se utiliza, cómo son las personas que trabajan en ciencia, en qué medida son responsables de los avances tecnológicos y cómo influyen en nuestra sociedad, etc. Tenemos, por tanto, un excelente recurso para incorporar conocimientos propios del enfoque CTS: evaluaciones sobre riesgos, efectos adversos, usos políticos, dilemas éticos o condicionamientos económicos de la investigación científica y el desarrollo tecnológico.

La Tabla 1 resume las películas que conforman la propuesta, con un breve detalle de la sinopsis y su temática general.

\begin{tabular}{|c|c|c|}
\hline PELÍCULA & SINOPSIS & TEMÁTICA \\
\hline $\begin{array}{l}\text { Victor } \\
\text { Frankenstein } \\
\text { (Paul McGuigan, } \\
\text { 2015) }\end{array}$ & $\begin{array}{l}\text { Igor Strausman, el asistente del doctor Victor } \\
\text { Frankenstein, intenta proteger al brillante } \\
\text { científico de sí mismo cuando pierde el } \\
\text { control con sus arriesgados experimentos y } \\
\text { vulnera los límites de lo ético y de lo racional. }\end{array}$ & $\begin{array}{l}\text { El uso irresponsable de } \\
\text { la tecnología, el control } \\
\text { de la naturaleza y la } \\
\text { dignidad humana. }\end{array}$ \\
\hline
\end{tabular}




\begin{tabular}{|c|c|c|}
\hline $\begin{array}{l}\text { Her } \\
\text { (Spike } \\
2013)\end{array}$ & $\begin{array}{l}\text { En Los Ángeles, un escritor desanimado } \\
\text { desarrolla una especial relación amorosa con } \\
\text { el sistema operativo de su computadora, una } \\
\text { intuitiva y sensible entidad llamada } \\
\text { Samantha. }\end{array}$ & $\begin{array}{llr}\text { La adicción } & \text { a } & \text { la } \\
\text { tecnología y y } & \text { sus } \\
\text { consecuencias } & \text { para } & \text { el } \\
\text { ser humano. } & & \end{array}$ \\
\hline $\begin{array}{l}\text { Avatar } \\
\text { (James Cameron, } \\
\text { 2009) }\end{array}$ & $\begin{array}{l}\text { Ambientada en el año } 2154 \text {, narra el conflicto } \\
\text { entre los Na'vi, habitantes del planeta } \\
\text { Pandora, y los humanos, debido a un } \\
\text { gigantesco árbol que cubre una inmensa veta } \\
\text { de un mineral muy cotizado y que supondría } \\
\text { la solución a los problemas energéticos de la } \\
\text { Tierra: el unobtainium. }\end{array}$ & $\begin{array}{lrr}\text { La lucha } & \text { por } & \text { los } \\
\text { recursos } & \text { energéticos } \\
\text { estratégicos } & \text { y la } & \text { crisis } \\
\text { ambiental. } & & \text { La } \\
\text { explotación de } & \text { los } \\
\text { pueblos originarios. }\end{array}$ \\
\hline $\begin{array}{l}\text { Lluvia } \text { de } \\
\text { albóndigas } \\
\text { (Philip Lord y } \\
\text { Chris Miller, } \\
\text { 2009) }\end{array}$ & $\begin{array}{l}\text { Flint Lockwood, un inventor fracasado, trata } \\
\text { de solucionar la falta de alimentos en su } \\
\text { pueblo con una máquina que hace caer } \\
\text { comida del cielo. Sin embargo, el aparato se } \\
\text { descontrola y amenaza con enterrar a todo el } \\
\text { mundo bajo grandes cantidades de comida. }\end{array}$ & $\begin{array}{l}\text { La imagen del } \\
\text { científico. ros } \\
\text { transgénicos y la ciencia } \\
\text { comorrárso } \\
\text { económico y político. }\end{array}$ \\
\hline $\begin{array}{l}\text { Walli-e } \\
\text { (Andrew Stanton, } \\
2008 \text { ) }\end{array}$ & $\begin{array}{l}\text { En el siglo XXI la producción excesiva de } \\
\text { basura que cubre la Tierra la ha hecho } \\
\text { inhabitable y la población es evacuada en } \\
\text { lujosas naves espaciales, mientras que un } \\
\text { ejército de robots compactadores de basura } \\
\text { llamados WALL-E (WasteAllocation Load } \\
\text { Lifter - Earthclass) se establecen en la Tierra } \\
\text { para restaurar y limpiar el planeta. } 700 \text { años } \\
\text { después, la última unidad WALL-E } \\
\text { superviviente encuentra una planta creciendo } \\
\text { entre la basura. }\end{array}$ & $\begin{array}{l}\text { El progreso tecnológico } \\
\text { y su riesgo para la salud } \\
\text { y el medio ambiente: } \\
\text { hiperconsumo, } \\
\text { tecnodependencia y } \\
\text { contaminación. }\end{array}$ \\
\hline $\begin{array}{l}\text { Yo, Robot } \\
\text { (Alex Proyas, } \\
2004)\end{array}$ & $\begin{array}{l}\text { En el } 2035 \text {, los robots forman parte de la vida } \\
\text { cotidiana y son la principal fuerza laboral de } \\
\text { la especie humana, trabajando con seguridad } \\
\text { gracias a las tres leyes de la robótica. Sin } \\
\text { embargo, el asesinato de un científico apunta } \\
\text { directamente hacia un sofisticado robot con } \\
\text { conciencia que pone en duda el cumplimiento } \\
\text { de las tres leyes. }\end{array}$ & $\begin{array}{l}\text { Tecnofilia y Tecnofobia. } \\
\text { Inteligencia artificial y } \\
\text { sus efectos en la vida } \\
\text { doméstica o las } \\
\text { relaciones laborales. }\end{array}$ \\
\hline $\begin{array}{l}\text { La máquina del } \\
\text { tiempo } \\
\text { (Wells, S, 2002). }\end{array}$ & $\begin{array}{l}\text { El científico Alexander Hartdegen ha } \\
\text { inventado una máquina con la que pretende } \\
\text { demostrar los viajes en el tiempo. Tas sufrir la } \\
\text { muerte de su prometida, decide huir al } \\
\text { pasado, pero un accidente provoca que la } \\
\text { máquina lo lleva al futuro, donde descubre } \\
\text { que la humanidad se ha dividido en dos clases } \\
\text { antagónicas: los elois, dulces, bellos e } \\
\text { inocentes, y los morlocks, monstruosos, } \\
\text { astutos y caníbales. }\end{array}$ & $\begin{array}{l}\text { Crítica social sobre el } \\
\text { destino de la especie } \\
\text { humana. Progreso } \\
\text { tecnológico, explotación } \\
\text { y alienación en el } \\
\text { capitalismo. }\end{array}$ \\
\hline $\begin{array}{l}\text { A.I } \\
\text { (Steven }\end{array}$ & $\begin{array}{l}\text { En el futuro, los seres humanos conviven con } \\
\text { robots humanoides llamados Mecas, pero }\end{array}$ & $\begin{array}{l}\text { La importancia de la } \\
\text { ética en la robótica y la }\end{array}$ \\
\hline
\end{tabular}




\begin{tabular}{|c|c|c|}
\hline Spielberg, 2001) & $\begin{array}{l}\text { cuando a un robot-niño llamado David se le } \\
\text { programa para tener sentimientos y amar a sus } \\
\text { padres adoptivos, se desatarán consecuencias } \\
\text { inesperadas para las que no están preparados. } \\
\text { David se verá solo en un mundo donde no } \\
\text { encaja ni entre máquinas ni entre humanos. }\end{array}$ & $\begin{array}{lr}\text { posibilidad de } & \text { que los } \\
\text { sistemas de inteligencia } \\
\text { artificial } & \text { sientan } \\
\text { emociones. }\end{array}$ \\
\hline $\begin{array}{l}\text { Matrix } \\
\text { (Wachowski, A., } \\
\text { y Wachowski, L., } \\
\text { 1999) }\end{array}$ & $\begin{array}{l}\text { Neo, un joven pirata informático descubre una } \\
\text { terrible realidad: los seres humanos son } \\
\text { usados por las máquinas para obtener energía, } \\
\text { y las pocas personas que han conseguido ser } \\
\text { liberadas viven de forma clandestina. Según } \\
\text { una profecía, Neo es el elegido para acabar } \\
\text { con las máquinas. }\end{array}$ & $\begin{array}{l}\text { Crítica a un mundo } \\
\text { hipertecnificado en el } \\
\text { que los seres humanos } \\
\text { han sido esclavizados } \\
\text { por las máquinas. }\end{array}$ \\
\hline $\begin{array}{l}\text { El gigante de } \\
\text { hierro (Brad Bird, } \\
\text { 1999) }\end{array}$ & $\begin{array}{l}\text { En 1957, un robot alienígena gigante es } \\
\text { descubierto por un niño. Entre ambos nace } \\
\text { una fuerte amistad que se verá amenazada } \\
\text { cuando el gobierno comienza a investigar los } \\
\text { hechos, en plena Guerra Fría. }\end{array}$ & $\begin{array}{l}\text { La carrera } \\
\text { armamentística y la la } \\
\text { amenaza constante de } \\
\text { guerra. Seguridad, } \\
\text { militarización y } \\
\text { mecanismos de control. }\end{array}$ \\
\hline $\begin{array}{l}\text { El hombre } \\
\text { bicentenario } \\
\text { (Chris Columbus, } \\
\text { 1999) }\end{array}$ & $\begin{array}{l}\text { En el siglo XXI, los robots son adquiridos } \\
\text { como electrodomésticos caseros, } \\
\text { especialmente programados para realizar } \\
\text { tareas menores. Uno de esos robots, Andrew, } \\
\text { comienza a experimentar emociones humanas } \\
\text { y a plantearse preguntas existenciales. } \\
\text { Entonces inicia un largo viaje de } \\
\text { autoconocimiento y lucha. }\end{array}$ & $\begin{array}{l}\text { Riesgos y temores que } \\
\text { la inteligencia artificial } \\
\text { despierta en los seres } \\
\text { humanos. } \\
\text { Transhumanismo y } \\
\text { singularidad. }\end{array}$ \\
\hline $\begin{array}{l}\text { Gattaca } \\
\text { (Andrew Niccol, } \\
\text { 1997) }\end{array}$ & $\begin{array}{l}\text { En el futuro, la mayor parte de los niños son } \\
\text { concebidos in vitro y con técnicas de } \\
\text { selección genética para garantizarles en el } \\
\text { éxito en la vida. Vincent, uno de los últimos } \\
\text { niños concebidos de modo natural, es } \\
\text { considero un ser inferior y, como tal, está } \\
\text { condenado a realizar los trabajos más } \\
\text { desagradables. Entonces, decide inventar un } \\
\text { plan complicado y peligroso en el que asume } \\
\text { ser alguien genéticamente superior. }\end{array}$ & $\begin{array}{l}\text { Nuevas formas de } \\
\text { discriminación basadas } \\
\text { en la información } \\
\text { genética. } \\
\text { totalitarismo científico y } \\
\text { la lucha por el libre } \\
\text { albedrío. }\end{array}$ \\
\hline $\begin{array}{l}\text { Contact } \\
\text { (Robert } \\
\text { Zemeckis, 1997) }\end{array}$ & $\begin{array}{l}\text { La científica Eleanor Arroway trabaja en el } \\
\text { programa SETI en busca de vida } \\
\text { extraterrestre. Su trabajo se ve recompensado } \\
\text { cuando detecta una señal extraterrestre que } \\
\text { contiene los planos de una máquina para } \\
\text { tomar contacto. Varias naciones del mundo } \\
\text { financian su construcción mientras se crea un } \\
\text { grupo internacional encargado de elegir al } \\
\text { representante de la humanidad para el } \\
\text { contacto. }\end{array}$ & $\begin{array}{l}\text { La búsqueda de vida } \\
\text { extraterrestre. La Big } \\
\text { Science. La vinculación } \\
\text { entre ciencia y empresa. } \\
\text { Las luchas de poder } \\
\text { entre empresas, ejércitos } \\
\text { y gobiernos. La relación } \\
\text { entre ciencia y religión. }\end{array}$ \\
\hline $\begin{array}{l}\text { La mosca } \\
\text { (David }\end{array}$ & $\begin{array}{l}\text { Un científico obsesionado con poder } \\
\text { conseguir la teletransportación diseña una }\end{array}$ & $\begin{array}{l}\text { La naturaleza de la } \\
\text { investigación científica }\end{array}$ \\
\hline
\end{tabular}




\begin{tabular}{|c|c|c|}
\hline $\begin{array}{l}\text { Cronenberg, } \\
1986)\end{array}$ & $\begin{array}{l}\text { máquina que prueba consigo mismo. Pronto } \\
\text { se dará cuenta de que una mosca también ha } \\
\text { sido expuesta a la máquina a la misma vez } \\
\text { que él y comienza a transformarse en mosca } \\
\text { gigante. }\end{array}$ & $\begin{array}{l}\text { y la experimentación. } \\
\text { Los riesgos de la } \\
\text { manipulación genética. }\end{array}$ \\
\hline $\begin{array}{l}\text { Nausicaä del } \\
\text { Valle del Viento } \\
\text { (Hayao Miyazaki, } \\
\text { 1984) }\end{array}$ & $\begin{array}{l}\text { En un futuro lejano, mil años después de una } \\
\text { guerra de carácter apocalíptico, los seres } \\
\text { humanos sobreviven a duras penas en las } \\
\text { cercanías de un bosque contaminado con } \\
\text { gases tóxicos e insectos mutantes gigantes, } \\
\text { que cubren gran parte de la Tierra. La } \\
\text { princesa Nausicaä se resiste a ver a los } \\
\text { insectos como enemigos. }\end{array}$ & $\begin{array}{l}\text { La naturaleza y la } \\
\text { supervivencia del medio } \\
\text { ambiente contra las } \\
\text { ansias de progreso } \\
\text { tecnológico y la codicia } \\
\text { humana. }\end{array}$ \\
\hline $\begin{array}{l}\text { Blade Runner } \\
\text { (Ridley Scott, } \\
\text { 1982) }\end{array}$ & $\begin{array}{l}\text { Rick Deckard es un Blade Runner, un agente } \\
\text { de policía destinado a cazar un grupo de } \\
\text { replicantes ilegales, los sofisticados NEXUS } \\
6 \text {, superiores en fuerza e inteligencia a los } \\
\text { humanos, pero diseñados para vivir una corta } \\
\text { existencia de cuatro años. Este grupo, que } \\
\text { anhela poder vivir más tiempo del que les han } \\
\text { adjudicado, fingen ser humanos para, } \\
\text { integrados en la sociedad, encontrar a su } \\
\text { creador y matarlo. }\end{array}$ & $\begin{array}{l}\text { La capacidad de crear } \\
\text { seres artificiales } \\
\text { idénticos a nosotros y } \\
\text { las nuevas formas de } \\
\text { esclavitud tecnológica. }\end{array}$ \\
\hline $\begin{array}{l}\text { Soylent Green } \\
\text { (Richard Fleisher, } \\
\text { 1973) }\end{array}$ & $\begin{array}{l}\text { En el año 2022, Nueva York cuenta con una } \\
\text { población de } 40 \text { millones de habitantes que } \\
\text { viven en condiciones miserables. Para } \\
\text { combatir el hambre se crea un alimento } \\
\text { sintético, el Soylent Green. Un detective } \\
\text { descubre que detrás del nuevo alimento hay } \\
\text { algo inquietante. }\end{array}$ & $\begin{array}{l}\text { La sobreexplotación y la } \\
\text { ruptura del equilibrio } \\
\text { ecológico. Eldesarrollo } \\
\text { tecnológico y su } \\
\text { relación con la política. }\end{array}$ \\
\hline $\begin{array}{l}\text { 2001: Odisea en } \\
\text { el espacio } \\
\text { (Stanley Kubrick, } \\
\text { 1968) }\end{array}$ & $\begin{array}{l}\text { Durante una misión de la NASA, la } \\
\text { supercomputadora HAL 9000, una máquina } \\
\text { dotada de inteligencia artificial, guía a un } \\
\text { equipo de tres astronautas en un viaje en el } \\
\text { que buscan descubrir los orígenes de la } \\
\text { humanidad. }\end{array}$ & $\begin{array}{l}\text { La creación de } \\
\text { inteligencias artificiales } \\
\text { pensantes. La tecnología } \\
\text { y sus efectos alienantes } \\
\text { sobre los humanos, }\end{array}$ \\
\hline $\begin{array}{l}\text { El hombre } \\
\text { invisible (James } \\
\text { Whale, 1933) }\end{array}$ & $\begin{array}{l}\text { Un científico experimenta sobre sí mismo un } \\
\text { suero para hacerse invisible. Su nueva } \\
\text { naturaleza lo transforma en un maníaco } \\
\text { homicida que quiere conquistar el mundo. }\end{array}$ & $\begin{array}{l}\text { Los límites éticos de la } \\
\text { experimentación y la } \\
\text { obligación del científico } \\
\text { de actuar conforme a un } \\
\text { código moral. }\end{array}$ \\
\hline
\end{tabular}

Con ánimo de ofrecer una lista lo más nutrida posible, se añaden estas otras aportaciones que pueden servir para seguir investigando las sociedades distópicas y su relación con la ciencia y la tecnología: 
-Metrópolis (Fritz Lang, 1927/1984)

-Nunca me abandones (Mark Romanek, 2010)

-Cuando el destino nos alcance (Richard Fleischer, 1973)

-Akira (Katsuhiro Ōtomo, 1988)

-Brazil (Terry Gilliam, 1985)

- Videodrome (David Cronenberg, 1987)

-Doce Monos (Terry Gilliam, 1996)

-Ready Player One (Steven Spielberg, 2018)

Sea cual sea la elección que se haga, los pasos que se siguen responden siempre a la misma estructura: Se dispone a los estudiantes en círculo o en forma de U para favorecer el diálogo y la atención ${ }^{22}$. La secuencia de actividades comienza con el visionado de una película o fragmento de película que será el núcleo sobre el que se va a trabajar. A continuación, se pide a los estudiantes que formulen alguna pregunta o cuestión que les haya sugerido. El propósito principal es partir de los propios intereses y preocupaciones del alumnado. Para ello, se pueden emplear diversas dinámicas de trabajo en función del número de alumnos. Por ejemplo, aplicar una estrategia de trabajo escalable que avance desde el diálogo en pequeño grupo hasta la puesta en común con el grupo completo ${ }^{23}$. Las preguntas se van escribiendo en la pizarra, indicando junto a ellas el nombre de la persona o el grupo que la formula. Los estudiantes deben tomar nota de todas las cuestiones en su cuaderno de clase. Estas preguntas se convierten entonces en el plan de trabajo o agenda de investigación, seleccionando aquella o aquellas que deseen comenzar a tratar. También aquí se pueden

\footnotetext{
${ }^{22}$ Si la distribución del aula no lo permite, se pueden aprovechar otros espacios del Centro, como la biblioteca o el aula de informática.

${ }^{23} \mathrm{La}$ virtud de esta estrategia es que permite que todos trabajen y tengan la oportunidad de dialogar, al menos con sus compañeros de grupo, aunque luego no tenga oportunidad de intervenir en el diálogo colectivo.
} 
emplear diversas estrategias de trabajo ${ }^{24}$. Con la primera pregunta elegida comienza el diálogo filosófico, siguiendo las pautas señaladas en la literatura especializada ${ }^{25}$. Una función importante del docente, transformado en mediador mayéutico, es mantener el equilibrio entre el plano formal y el plano material. El primero hace referencia al arte de la argumentación filosófica, exigiendo claridad y pertinencia en las intervenciones, así como identificando fortalezas y debilidades en las argumentaciones. El segundo implica reconocer las grandes cuestiones filosóficas que surgen en el diálogo, reformulando los discursos de los estudiantes y estableciendo relaciones con los autores y sus obras. Además, como lo que se pretende es que el intercambio de opiniones sea cada vez más complejo, crítico y reflexivo, las sesiones siguientes se pueden organizar en torno a la clarificación de uno o varios conceptos, la búsqueda de documentación sobre un problema o la discusión de una controversia. Finalmente, los estudiantes elaboran una disertación de tema libre en la que tienen que demostrar su capacidad de investigar sobre una controversia tecnocientífica, argumentar y contraargumentar desde las distintas posturas enfrentadas, así como exponer y defender su opinión personal. La presentación de las disertaciones se acompañará con la defensa oral de los resultados. Además, se animará a la utilización de soportes informáticos.

Todas estas estrategias están encaminadas a la elaboración de productos "socialmente relevantes" donde el alumnado pueda comprometerse activamente en la transformación de su entorno: diseño de blogs o páginas web; colaboraciones en la revista escolar; organización de actividades de integración o coeducación;

\footnotetext{
${ }^{24}$ La votación a mano alzada conlleva el riesgo de que la elección de la pregunta sea cooptada por las relaciones de liderazgo, o de imponer una mayoría.

${ }^{25}$ A modo de ejemplo, se pueden mencionar las obras de Oscar Brenifier, o Michel Tozzi y su esquema de las tres fases del diálogo filosófico: problematización, conceptualización y argumentación.
} 
preparación de actividades para las Jornadas Culturales del Centro; planificación y desarrollo de campañas de participación ciudadana simulada o real- o campañas de publicidad para difundir valores éticos, etc. De este modo, el alumnado aparece como un agente activo, protagonista y corresponsable de su propio aprendizaje. Es decir, se sugiere un amplio abanico de prácticas y hábitos por parte del alumnado que muchas veces se descuidan desde planteamientos más contemplativos, pero que no deja fuera ningún aspecto de lo que hemos definido como una cultura científica crítica y responsable.

Con la intención de complementar el marco teórico para una educación STEAM \& Ethics tal y como se ha ido describiendo en las líneas precedentes, se ofrece un ejemplo práctico que ilustra la secuencia de actividades a seguir.

\section{Propuesta de trabajo en el aula}

Para ejemplificar una sesión práctica en el aula de "Valores éticos", $1^{\circ}$ Ciclo de ESO, en el bloque temático correspondiente a "los valores éticos y su relación con la ciencia y la tecnología" se ha escogido el visionado de la película "Lluvia de albóndigas" (Philip Lord y Chris Miller, 2009), dedicada a la temática sobre manipulación genética. En concreto, las cuestiones relacionadas con los alimentos genéticamente modificados y sus efectos para la salud y el medio ambiente constituyen un caso típico de los estudios CTS, ya que ponen de manifiesto la complejidad del desarrollo científico-tecnológico donde se hacen explícitos los aspectos éticos, políticos o económicos y no solo los estrictamente técnicos.

Actividad 1: Visionado de "Lluvia de albóndigas" 
En concreto se han seleccionado dos fragmentos. En el primer fragmento (minutos 00:48/04:41-20:33) se presenta al protagonista, Flint Lockwood, un joven científico que sueña con inventar algo que mejore la vida de todos los habitantes de su ciudad: una máquina que convierte el agua en comida. La máquina es enviada a las nubes y comienza a caer comida del cielo. Al principio, la comida climática es un éxito querido por todos: la chica del tiempo Sam Sparks se queda en el pueblo para cubrir lo que ella llama "el mayor fenómeno meteorológico de la historia". El alcalde ve la oportunidad de lograr sus aspiraciones políticas y rescatar a la ciudad de la crisis. Los ciudadanos pueden disfrutar por fin de un variado menú.

En el segundo fragmento (minutos 39:43-51:24) la máquina se descontrola y comienza a arrojar toneladas de comida provocando una gran catástrofe en la ciudad. El protagonista y sus amigos deberán arreglar la máquina para que todo vuelva a la normalidad. De este modo, el primer fragmento recoge las diferentes formas en que la actividad científico-tecnológica interactúa con la sociedad en tanto que recurso económico y político, mientras que el segundo muestra los beneficios y los riesgos del desarrollo científicotecnológico.

Tras el visionado, se pide a los estudiantes que formulen alguna pregunta o cuestión que les haya sugerido. Con la primera pregunta elegida comienza el diálogo filosófico.

\section{Actividad 2: Pautas para el diálogo}

Una vez que hemos conseguido el requisito de fomentar el interés y la motivación del alumnado, centrando su atención en una pregunta que es de su interés, el paso siguiente consiste en crear un espacio de intercambio verbal y colectivo de ideas en el que el alumnado aprenda a ensayar aquellas habilidades de pensamiento que resultan imprescindibles para afrontar los problemas de un mundo 
continuamente transformado por el desarrollo científicotecnológico.

Durante el diálogo, el docente puede ofrecer preguntas-guía para orientar y ayudar al intercambio de opiniones. Por ejemplo:

-¿Crees que el protagonista, Flint, está loco? ¿Por qué? ¿Cómo lo definirías?

-¿Cuáles son los rasgos que debe tener un científico?

-¿Por qué nadie en la ciudad quiere a Flint? ¿Qué hace que eso cambie?

-Flint hace realidad su deseo y le sale mal ¿Por qué le paso eso?

$-¿$ Crees que es una situación posible?

-¿Qué habría pasado si la máquina de Flint no se hubiese descontrolado?

-¿Cómo crees que se podría haber evitado el desastre?

¿¿De qué otra manera podría haber terminado la historia?

-¿Tiene Flint alguna responsabilidad de lo ocurrido?

- ¿Tienen los habitantes de la ciudad alguna responsabilidad?

-En tu opinión, ¿Quién es el principal responsable de lo ocurrido?

$-¿$ Crees que los protagonistas de la historia aprenden alguna lección? ¿Cuál?

-¿Todo lo que se puede hacer tecnológicamente, se debe hacer?

-¿Es lo mismo progreso tecnológico que progreso humano?

-En una situación de controversia científico-social ¿Qué opiniones deberían ser tenidas en cuenta?

-¿Qué papel podemos jugar cada uno de nosotros?

Es importante dejarles que opinen, que imaginen, formulen hipótesis, busquen alternativas, anticipen consecuencias o seleccionen posibilidades, preguntando constantemente por las razones que tienen para apoyar sus opiniones. Aquí, los estudiantes pueden dar todo tipo de respuestas. De ahí la importancia de 
distinguir entre razonamientos mejores y peores, buenas y malas razones, opiniones y decisiones más o menos razonadas, etcétera.

\section{Actividad 3: Análisis de una noticia}

Por citar un ejemplo sobre la actualidad de estos temas, hace unas pocas semanas 25.000 científicos procedentes de 127 institutos de investigación de toda Europa se unieron para dirigir una carta abierta al Parlamento Europeo, la Comisión Europea y el Consejo, exigiendo un cambio urgente en la legislación europea que equipara las plantas modificadas con CRISPR con los transgénicos convencionales (OMG). Esta decisión deja a Europa fuera de juego ante la posibilidad de usar CRISPR para diseñar nuevas variedades vegetales resistentes a la sequía y que necesiten menos agua y pesticidas. En un contexto de cambio climático, el texto alega que "la capacidad de usar la edición genética es crucial para el bienestar y la seguridad alimentaria de los ciudadanos europeos" ${ }^{26}$.

Se trata, en definitiva, de abordar, con muchos matices, la controversia entre los defensores y detractores de los alimentos genéticamente modificados. Para ello, se parte del análisis de una noticia, reportaje o cualquier otro documento que plantee en formato periodístico una controversia científica en la que estén implicados varios actores sociales con ideas, opiniones e intereses diversos. Científicos, ingenieros, empresas, políticos, ecologistas, asociaciones profesionales, ciudadanos, organizaciones de la sociedad civil, etc., pueden constituir esa red de actores.

El análisis del documento puede incluir desde la definición precisa de los conceptos científicos que aparecen en él -por ejemplo, definir y explicar qué son los alimentos transgénicos, historia y

${ }^{26}$ DOMÍNGUEZ, Nuño: "Los científicos se rebelan contra la ley europea de transgénicos", El Pais, Madrid, 2019. Disponible en https:/elpais.com/elpais/2019/08/07/ciencia/1565191737_505932.html (último acceso 19/09/2019) 
principales usos, ventajas y desventajas, etc.- hasta la elaboración de una disertación en la que cada uno de los estudiantes tenga que argumentar y contraargumentar desde las distintas posturas enfrentadas, así como exponer y defender en público su opinión personal, usando como apoyo una presentación en PowerPoint $\mathrm{u}$ otro programa de presentaciones digitales. Para acotar el abordaje de la disertación, se puede suministrar el siguiente cuestionario:

-¿Qué sabes de los OMG? Describe un poco en qué consisten.

-¿Qué alimentos conoces que sean OMG?

-¿Qué efectos conoces de los OMG en la salud de las personas?

-¿Existe algún riesgo para nuestra salud? ¿Podemos asumirlo?

-¿Cómo crees que afecta el cultivo de los OMG a los cultivos convencionales?

¿¿Crees que hay algún uso adecuado? ¿Cuál podría ser?

- ¿Se debería prescindir de los OMG en la actualidad? ¿Se podría?

-En ambos casos, ¿qué ventajas e inconvenientes tiene esta decisión?

-Con la información disponible y la que hayáis buscado en vuestra investigación, y en coherencia con las respuestas a las cuestiones anteriores, ¿Crees que la legislación europea debe ser modificada?

-En caso afirmativo, ¿qué alternativas se propondrían?

-¿Puedes identificar a los distintos actores involucrados? ¿A quién afecta la legislación?

-¿Hay otros aspectos que se deban considerar para tomar una decisión razonada?

-¿Quién crees que debería decidir?

También se pueden proporcionar documentos polémicos relacionados directa o indirectamente con la noticia inicial y que puedan ser utilizados como informaciones complementarias por los estudiantes. Por ejemplo: 
-El reportaje de Jordi Pérez Colomé “¿Pueden los transgénicos salvar el planeta?" Disponible en https://elpais.com/elpais/2016/11/13/eps/1478991954_147899. html

-El artículo de opinión de Juan Echanove (2015): "Transgénicos: medias verdades y grandes mentiras". Disponible

en https://elpais.com/elpais/2016/07/18/ciencia/1468859244_6864 47.html

-La entrevista al biólogo molecular Richard J. Roberts (2017).

Disponible

https://elpais.com/elpais/2017/07/04/ciencia/1499183349_9151

92.html

Este ejercicio permite trabajar las distintas formas de expresar el pensamiento a través del lenguaje. Resumir, narrar y describir son habilidades de gran importancia ya que tienen que ver con la organización de la información, y nos ayudan a pensar $\mathrm{y}$ seleccionar las ideas esenciales, expresándolas de manera ordenada.

\section{Actividad 4: Investigación-acción}

No tiene sentido una formación para la ciudadanía democrática que no incluya el aprendizaje de la participación en decisiones relacionadas con el cambio social y el desarrollo científicotecnológico. A participar se aprende participando. Por ello, el objetivo de esta última actividad es incorporar en el aula procesos de toma de decisiones contextualizadas en los problemas del presente y del futuro. En concreto, se ha escogido la discusión de una controversia en la que el valor de la información y los argumentos, la responsabilidad social y ambiental, la voluntad de negociar los disensos y de consensuar las decisiones, sean algunos de los aprendizajes más importantes. 
Tras el análisis de la noticia y la identificación de los principales actores afectados e implicados en la controversia de los alimentos genéticamente modificados, se crean diferentes grupos de trabajo colaborativo para cada uno de los actores sociales, de modo que cada grupo tenga que definir el perfil de su actor social asignado y buscar información que le permita defender su postura en la controversia.

Se trata, en definitiva, de buscar información de diferentes fuentes (informes oficiales, entrevistas, artículos de opinión, blogs, etc.) para poner de manifiesto las diversas razones, intereses, valores y motivaciones presentes en la polémica.

Una vez realizada la investigación en colaboración grupal, los distintos actores sociales deben exponer sus posturas de forma argumentada y ordenada racionalmente, utilizando para ello medios informáticos y audiovisuales. Una vez considerados todos los puntos de vista, se tendrá que consensuar una solución final a la controversia. Este último proceso permite una gran flexibilidad, pudiendo diseñar acciones que integren varias áreas o materias. Por ejemplo, crear una página web, iniciar una campaña de publicidad, organizar una recogida de firmas, etc., con el fin de atraer la atención sobre una postura o movilizar a un determinado colectivo. Una de las virtudes de esta estructura es que permite una gran flexibilidad, pudiendo adaptarse a diferentes niveles y contextos curriculares, así como a distintas temporalizaciones. Quede esta presentación como una invitación hacia una educación STEAM \& Ethics que busca promover una cultura científica más crítica y responsable, acorde con los retos del futuro.

\section{Consideraciones finales}

A estas alturas, ya nadie duda del papel central de la actividad científica y tecnológica en ámbitos como la producción de bienes y 
servicios, los flujos de información y comunicación, la transformación de la naturaleza e incluso de las formas de comportamiento y de organización social y política. Esta situación conlleva un número cada vez más amplio de problemas relacionados con la ciencia y la tecnología que involucran aspectos políticos, económicos y éticos, principalmente. Como consecuencia, nos vemos abocados, de manera creciente, a hacer uso de la reflexión crítica para poder tomar decisiones fundadas como consumidores en un supermercado, como usuarios de un sistema de salud, como padres, como empresarios o como trabajadores.

En el terreno educativo, son muchas las voces que enfatizan la necesidad de formar futuros ciudadanos y profesionales que aúnen una valoración global positiva del desarrollo científico $\mathrm{y}$ tecnológico al tiempo que desenvuelvan herramientas éticas de responsabilidad social. Los nuevos desafíos del mundo contemporáneo en materia de alimentación, salud, innovación o medioambiente requieren, por tanto, de enfoques renovados para una educación integral. La propuesta de una educación STEAM \& Ethics tal y como se ha defendido en este trabajo refleja esa convicción e introduce una transformación conceptual y práctica que apunta al desarrollo del pensamiento crítico, la reflexión ética y el compromiso social.

Sin embargo, en el marco español, la evolución de las últimas tres materias que se han diseñado para el fomento de la cultura científica muestra un panorama preocupante, disminuyendo progresivamente las referencias a la sociedad, la ética, la participación ciudadana o la democracia. De ahí la necesidad de crear nuevos espacios curriculares donde poder ensayar nuevas estrategias y recursos didácticos para conseguir el reto de una cultura científica crítica y responsable.

Consciente del reto que ello supone, el objetivo de este trabajo ha sido presentar una propuesta didáctica para el aula de "Valores 
éticos" del $1{ }^{\circ}$ Ciclo de ESO, en el bloque temático correspondiente a "los valores éticos y su relación con la ciencia y la tecnología". Los estudios CTS y la metodología de FpN han sido los fundamentos teóricos y prácticos de la propuesta. La combinación de ambos enfoques está en condiciones de avanzar hacia un nuevo modelo de educación STEAM \& Ethics.

Quizá la mayor contribución política de los estudios CTS sea la promoción de la participación ciudadana. De acuerdo con el llamado "silogismo CTS"27: en la medida en que el desarrollo científico-tecnológico se ha convertido en un asunto público de primera magnitud, las sociedades democráticas que comparten el compromiso básico de participación ciudadana en los asuntos públicos deberían promover esa participación social y ciudadana en los temas relacionados con la ciencia y la tecnología.

Ahora bien, esa socialización democrática depende en gran medida de la práctica, de ahí que sea necesario que los estudiantes tengan la oportunidad de familiarizarse con el gusto por la pregunta y la investigación, la búsqueda crítica de información, la argumentación, la sensibilidad ética y la creatividad en la resolución de problemas. Para ello se considera que el método más adecuado es el diálogo realizado en una comunidad que busca, trabaja e investiga conjuntamente. En este sentido, la metodología de FpN es considerada la más idónea para promover la cultura científica desde una perspectiva CTS, ya que posibilita un aprendizaje significativo, estimulando la reflexión y ayudando al cuestionamiento y replanteamiento de todos aquellos temas y asuntos que pueden preocupar al alumnado. No se trata ni de una asimilación y apoyo acrítico de todo lo relacionado con la ciencia y la tecnología, ni de una crítica global infundada, sino de facilitar que los estudiantes aprendan a expresar sus opiniones, a hablar y a

${ }^{27}$ GONZÁLEZ GARCÍA, Marta, LOPEZ CEREZO, José Antonio y LUJÁN, José Luis: Ciencia, tecnología y sociedad: una introducción al estudio social de la ciencia y la tecnología, Tecnos, Madrid, 1996. 
escucharse, ayudándoles a encontrar razones mejores y más sólidas para creer en aquellas cosas en las que, después de una cuidadosa reflexión, han elegido creer.

En la búsqueda de recursos que fomenten el diálogo filosófico, el uso del cine de ciencia-ficción aparece como una excelente estrategia didáctica para trabajar diversos aspectos ligados al mundo propio del alumnado y motivar la discusión y el intercambio de opiniones. El cine constituye no solo uno de los principales medios de transmisión de los valores y patrones culturales de una sociedad, las creencias compartidas, las ideologías, las utopías y las distopías, sino también uno de los entretenimientos de mayor interés e influencia en la vida de los adolescentes, siendo la ciencia ficción uno de los géneros cinematográficos más populares. Así, por un lado cumple con el requisito de fomentar actitudes positivas entre los estudiantes más jóvenes, fuertemente atraídos por los mundos imaginarios que se reflejan en las películas, mientras que por otro lado motiva la reflexión sobre la visión del mundo científico que transmite, animándoles a establecer puentes entre los contenidos fílmicos y la realidad, como el análisis de la imagen de la ciencia y los científicos, los riesgos y beneficios de la ciencia y la tecnología en el futuro y la responsabilidad social del científico y la ciudadanía en general. Para ello se han seleccionado algunas de las películas y fragmentos de películas con más contenido CTS. Además, siguiendo las recomendaciones de la normativa vigente, se ha incorporado a la propuesta original de FpN la realización de tareas, proyectos o situaciones-problema que el alumnado debe resolver haciendo uso de sus conocimientos y habilidades. Esta metodología pretende ayudar al alumnado a asumir la responsabilidad de su aprendizaje, ejerciendo la tarea investigadora y aplicándola a proyectos reales. Se favorece, por tanto, un aprendizaje orientado a la acción en el que se integran distintos tipos de conocimientos, destrezas, actitudes y valores personales, es decir, los elementos que integran las distintas competencias. 
Así, por ejemplo, el diálogo filosófico en una comunidad de investigación colectiva contribuye al desarrollo de la competencia en comunicación lingüística (CL) al plantear situaciones-problema en las que necesariamente debemos compartir aquello que percibimos, sentimos o pensando, mejorando constantemente nuestro lenguaje para ser adecuadamente entendidos por el otro. En ese movimiento dialógico se atiende también a las competencias sociales y cívicas (CSC), ya que implica simultáneamente actitudes de respeto y tolerancia a las posiciones contrarias. El respeto a los valores sociales y cívicos se convierte en la práctica habitual del aula. Además, en la medida en que los problemas filosóficos abordados forman parte de la herencia cultural del alumnado, y se fomenta la curiosidad y el interés hacia otras manifestaciones culturales relacionadas con la filosofía -como el cine-, las actividades programadas también promueven las competencias relacionadas con la conciencia y las expresiones culturales (CEC). Por otro lado, la incorporación de actividades de resolución de situaciones-problema como, por ejemplo, controversias científicas que requieren, para la toma de decisiones, tareas de investigación, análisis, argumentación, emisión de juicios fundados, etc., promueve la adquisición de destrezas vinculadas a las competencias básicas en ciencia y tecnología (CMCT). Finalmente, las competencias relacionadas con el sentido de la iniciativa y espíritu emprendedor (SIEE), la competencia digital (CD) o la consolidación de hábitos de trabajo y estudio propios de la competencia aprender a aprender (AA) se movilizan a la hora de buscar información en distintas fuentes, preparar una presentación en soporte informático o crear productos finales encaminados a una solución práctica.

En definitiva, se considera que la nueva propuesta de una educación STEAM \& Ethics, basada en la combinación del enfoque CTS y la metodología dialógica de FpN, no solo atiende a las recomendaciones de la normativa vigente sino que está en 
condiciones de promover ciudadanos científicamente cultos, críticos y responsables respeto al cambio científico-tecnológico, que se forman una opinión sobre las potencialidades y los riesgos de la ciencia y la tecnología, que se involucran en los procesos de toma de decisiones y que son capaces de alcanzar un consenso sobre las mismas.

No obstante, el presente trabajo se ha limitado a sugerir solo algunas pautas de trabajo. Los posibles usos didácticos pueden ser variados, adaptarse a diferentes niveles y contextos educativos, así como a distintas áreas o materias. La propuesta didáctica que se ha ejemplificado pretende servir como base para proporcionar ideas y recursos a aquellos profesionales de la educación que se interesen por actividades en el aula basadas en el diálogo y el recurso al cine de ciencia-ficción. Quede esta propuesta como una invitación para conocer una aproximación a los temas de ciencia y tecnología amplia y compleja, pero también más atractiva.

\section{Bibliografía}

AMAR, Víctor: Comprender y disfrutar el cine. La gran pantalla como recurso educativo, Grupo Comunicar, Huelva, 2003.

CABERO, Julio: "Educación en valores y cine", en Making of: Cuadernos de Cine y Educación, N. ${ }^{\circ}$ 20, Barcelona, 2003. Págs.1630.

CÁMARA HURTADO, Montaña y LÓPEZ CEREZO, José Antonio: "Dimensiones políticas de la cultura científica, en LÓPEZ CEREZO, José Antonio y GÓMEZ GONZÁLEZ, Francisco Javier: Apropiación social de la ciencia, Biblioteca Nueva-OEI, Madrid. Págs. 63-89.

DÍAZ, Irene y GARCÍA, Myriam: "Más Allá del Paradigma de la Alfabetización. La Adquisición de Cultura Científica como Reto Educativo", en Formación Universitaria, Vol. 4 N. ${ }^{\circ}$ 2, Chile, 2011. 
Págs. 3-14.

GONZÁLEZ, Marta, LÓPEZ CEREZO, José Antonio y LUJÁN, José Luís: Ciencia, tecnología y sociedad: una introducción al estudio social de la ciencia y la tecnología, Tecnos, Madrid, 1996. LIPMAN, Matthew, SHARP, Ann y OSCAYÁN, Frederick: La filosofía en el aula, Ediciones de la Torre, Madrid, 1992.

LIPMAN, Mathew: Pensamiento complejo y educación, Ediciones de la Torre, Madrid, 1998.

MARTÍN GORDILLO, Mariano: "Espacios curriculares para una ciencia cordial", en MARTÍN GORDILLO, Mariano y MARTINS, Isabel: Ciencia cordial. Un desafio educativo, Los Libros de la Catarata, Madrid, 2018.

MECD: Anuario de Estadísticas Culturales 2018, Secretaría General Técnica, Subdirección General de Atención al ciudadano, Documentación y Publicaciones, MECD, Madrid, 2018.

MECD: Orden ECD/65/2015, de 21 de enero, por la que se describen las relaciones entre las competencias, los contenidos y los criterios de evaluación de la Educación Primaria, la Educación Secundaria Obligatoria y el Bachillerato, MECD, Madrid, 2015. MECD: Real Decreto 1105/2014, de 26 de diciembre, por el que se establece el currículo básico de la Educación Secundaria Obligatoria y del Bachillerato, Ministerio de Educación, Cultura y Deporte, Madrid, 2014.

MECD: Ley Orgánica 8/2013, de 9 de diciembre, para la Mejora de la Calidad Educativa, Ministerio de Educación, Cultura y Deporte, Madrid, 2013.

PARLAMENTO EUROPERO Y CONSEJO DE LA UNIÓN EUROPEA: Recomendación 2006/962/CE del Parlamento Europeo y del Consejo, de 18 de diciembre de 2006, sobre las competencias clave para el aprendizaje permanente, Diario Oficial de la Unión Europea L 394/10, Bruselas, 2006.

SOUSA, David y PILECKI, Tom: From STEM to STEAM: Using Brain-Compatible Strategies to Integrate the Arts, CA: SAGE, 
Thousand Oaks, 2013.

TEZANOS, José Félix: "Los impactos sociales de la revolución tecnológica", en TEZANOS, José Félix: Los impactos sociales de la revolución científico-tecnológica, Noveno Foro sobre Tendencias Sociales, Sistema, Madrid, 2007. Págs. 31-62. 



\section{COMENTARIOS, INFORMES Y ENTREVISTAS}

\section{STUDIES, REPORTS AND INTERVIEWS}


